\title{
Quad channel software defined receiver for passive radar application
}

\author{
TAMÁs Pető, Rudolf SEller \\ Department of Broadband Infocommunication and Electromagnetic Theory \\ Budapest University of Technology and Economics \\ Egry József street 18, 1111 Budapest, Hungary \\ e-mail:peto@hvt.bme.hu,seller@hvt.bme.hu
}

(Received: 08.12.2015, revised: 19.07.2016)

\begin{abstract}
In recent times the growing utilization of the electromagnetic environment brings the passive radar researches more and more to the fore. For the utilization of the wide range of illuminators of opportunity the application of wideband radio receivers is required. At the same time the multichannel receiver structure has also critical importance in target direction finding and interference suppression. This paper presents the development of a multichannel software defined receiver specifically for passive radar applications. One of the relevant feature of the developed receiver platform is its up-todate SoC (System on hip) based structure, which greatly enhance the integration and signal processing capacity of the system, all while keeping the costs low. The software defined operation of the discussed receiver system is demonstrated with using DVB-T (Digital Video Broadcast - Terrestrial) signal as illuminator of opportunity. During this demonstration the multichannel capabilities of the realized system are also tested with real data using direction finding and beamforming algorithms.
\end{abstract}

Key words: DPIS, multichannel receiver, passive radar, PCL, SDR

\section{Introduction}

Passive radars take advantage of already existing radio sources to detect targets. Researches have investigated the potential illuminators of opportunity such as the FM radio, GSM, UMTS, Wi-Fi, WiMAX, DAB, DVB-T, DVB-S signals. The basic proof of concept has been verified extensively so far for the different illuminator signals [4-6]. Software defined radio platforms are commonly used in a passive radar demonstrator system due to the flexibility of such receiver structures $[1-3,10]$. The application of multiple IOPs (Illuminator of Opportunity) located in different bands at the same time is also a field of interest in high range resolution and imaging application [10]. However these modular systems are very high-priced and inhibit the dissemination of passive radar technology. Low cost software defined receivers that are currently available in wide range are also investigated for passive radar application, how- 
ever the high dynamic range and precise multichannel operation is not achievable with these units.

One of the most significant limitation factor of passive radars is the direct path interference [6].The suppression of the strong direct path reference signal in the surveillance channel is essential in order to achieve a reasonable signal-to-noise ratio in large bistatic target distances. One of the most straightforward solution for this issue is the use of spatial filtering with beamforming algorithms [7-9]. FM radio based beamforming passive radars have been developed successfully in recent times [8].

In this paper we describe our developed multichannel passive radar receiver structure which is based on SoC (System on Chip) architecture. Our approach not only reduces the cost of the system, but increases the system integration and provides an up-to-date high performance platform for passive radar signal processing. The demonstration of basic spatial filtering implementation on the designed system is also presented. The design considerations (Section 2), the structure (Section 3) and the results (Section 4) of the developed hardware platform are described in detail. The effective operation of the platform is tested via field measurements.

\section{Design considerations}

The required parameters of the receiver system are fundamentally dependent on the properties of the used illuminator signals. The receiver must be able to cover sufficiently high dynamic range to the simultaneous reception of the high power direct path signal and the weak target echoes. At the same time the bandwidth of the used reference signal is also significant to achieve fine range resolution. For a given illuminator signal the achievable bistatic range resolution can be calculated using Eq. (1). In Eq. (1) $c$ stands for the speed of light, $B$ denotes the bandwidth of the signal and $\beta$ denotes the bistatic angle.

$$
\Delta_{r}=\frac{c}{2 B \cos \left(\frac{\beta}{2}\right)} .
$$

Table 1 summarizes the main parameters of the most common illuminators of opportunity. To determine a real requirement for the dynamic range of the receiver system, the power level of the direct path signal has been calculated for the different applicable illuminators using Eq. (2) with the baseline distance of $L=10 \mathrm{~km}$. (Antennas with $0 \mathrm{dBi}$ gain are assumed both on the transmitter and the receiver side.)

$$
P_{\text {direct }}=\frac{\left(P_{t} \lambda^{2}\right)}{(4 \pi)^{2} L^{2}},
$$

where $P_{t}$ is the power of the transmitter, $\lambda$ denotes the wavelength of the IOP and $L$ denotes the distance from the reference transmitter. In order not to lose sensitivity because of digita- 
lization, the quantization noise of the ADC (Analog Digital Converter) must be lower than the thermal noise floor for the given illuminator bandwidth plus the external noise. The thermal noise floors have been calculated with Eq. (3).

$$
P_{n}=k T_{s} B,
$$

where $k$ is the Boltzmann constant, $T_{s}=300 \mathrm{~K}$ is the system temperature and $B$ denotes the bandwidth of the signal. The power of the external noise that is originating from man-made or natural sources can be estimated using the ITU-R [P.372-8] recommendation. This recommendation is intended to use for system performance determinations and design. The external noise figure denoted with $F_{a}$ expresses the power of the external noise above thermal noise with reference temperature of $300 \mathrm{~K}$ (our assumed system temperature). For frequencies bellow $1 \mathrm{GHz}$ the values indicated in Table 1 belong to the curve of the man-made noise in business environment. In frequencies above $\sim 1 \mathrm{GHz}$ noise from individual sources such as galactic noise, atmospheric gasses, etc. have usually less noise temperature than $300 \mathrm{~K}$ (Expect the noise of the Sun).

Table 1. Relevant parameters of the most common illuminators

\begin{tabular}{l|c|c|c|c|c|c}
\hline Illuminator & EIRP & Frequency & Bandwidth & $\begin{array}{c}\text { Received power } \\
(\boldsymbol{L}=\mathbf{1 0} \mathbf{~ k m})\end{array}$ & $\begin{array}{c}\text { Thermal } \\
\text { noise } \\
\text { floor }\end{array}$ & $\begin{array}{c}\text { External } \\
\text { noise factor }\end{array}$ \\
\hline $\mathrm{FM}$ & $100 \mathrm{~kW}$ & $\sim 100 \mathrm{MHz}$ & $\sim 75 \mathrm{kHz}$ & $-12 \mathrm{dBm}$ & $-125 \mathrm{dBm}$ & $22 \mathrm{~dB}$ \\
\hline $\mathrm{GSM}$ & $100 \mathrm{~W}$ & $900,1800 \mathrm{MHz}$ & $200 \mathrm{kHz}$ & $-61,-67 \mathrm{dBm}$ & $-120 \mathrm{dBm}$ & $8 \mathrm{~dB},<0$ \\
\hline $\mathrm{UMTS}$ & $100 \mathrm{~W}-1 \mathrm{~kW}$ & $2100 \mathrm{MHz}$ & $3.84 \mathrm{MHz}$ & $-59 \mathrm{dBm}$ & $-108 \mathrm{dBm}$ & $<0$ \\
\hline $\mathrm{DAB}$ & $10 \mathrm{~kW}$ & $\sim 220 \mathrm{MHz}$ & $1.536 \mathrm{MHz}$ & $-29 \mathrm{dBm}$ & $-112 \mathrm{dBm}$ & $13 \mathrm{~dB}$ \\
\hline $\mathrm{DVB}-\mathrm{T}$ & $1-100 \mathrm{~kW}$ & $\sim 600 \mathrm{MHz}$ & $7.61 \mathrm{MHz}$ & $-28 \mathrm{dBm}$ & $-105 \mathrm{dBm}$ & $9 \mathrm{~dB}$ \\
\hline $\mathrm{DVB}-\mathrm{S}$ & $100-1000 \mathrm{~kW}$ & $10.7-12.7 \mathrm{GHz}$ & $27-30 \mathrm{MHz}$ & - & $-99 \mathrm{dBm}$ & $<0$ \\
\hline
\end{tabular}

Having examined the parameters of different illuminators the analog digital converter of the receiver system must have at least $\sim 70 \mathrm{~dB}$ dynamic range in order to reduce SNR (Signal-to-Noise Ratio) degradation originating from the quantization noise. Thus for the digital conversation we are using 12 bit wide $\mathrm{ADC}$ with $100 \mathrm{MHz}$ sampling frequency providing slightly more than $70 \mathrm{~dB}$ SNR. The effective number of bits and thus the SNR for illuminator signals with small bandwidth can be further increased with performing integration in the decimation stage.

Nowadays several research groups deal with using the DVB-T signal in passive radars. From among the potentially applicable illuminators of opportunity, the DVB-T signal not only has beneficial correlation properties but also has relatively wide $7.61 \mathrm{MHz}$ bandwidth and high power transmitters. Thus it was expedient to test the capabilities of the receiver with this type of illuminator. The demonstration results are presented in Section 4. 


\section{Receiver structure}

The block diagram of the designed receiver platform is illustrated in Fig. 1 and Fig. 2.

\subsection{RF front-end}

RF signals coming from the antenna system are first received by the front-end unit. This unit is responsible for down-mixing the RF signal to the intermediate frequency which can be processed further by the digital data acquisition card. Fig. 1 shows the detailed architecture of the RF signal path.

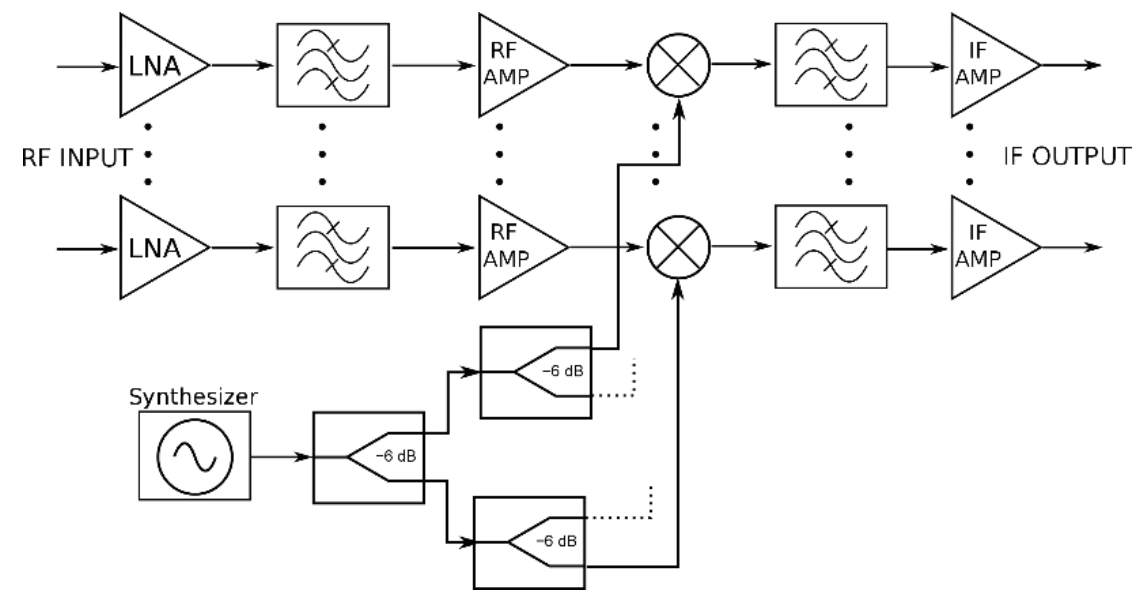

Fig. 1. Block diagram of the multichannel coherent RF receiver

The first stage of the RF front-end unit is a low noise amplifier which is responsible for achieving a low noise figure for the overall system. After the first stage the signal is filtered by an optional band pass filter. Application of the RF filter can improve the out-of-band interference tolerance capability of the system, but also limits the available illuminators for the receiver. For the demonstration presented in Section 4 a filter designed to operate in the DVB-T band has been utilized (460 MHz $-800 \mathrm{MHz}$ ). In order to maintain the proper signal level in the entire RF chain a second RF amplifier has been placed before the signal is mixed down to the IF (Intermediate Frequency) band. It is also important that in passive radar scenarios the reference signal often has very high power at the receiver side, thus the system must also handle the these signals. Beside this the amplifier stage must have high linearity to avoid the appearance of the undesired harmonic components in the spectrum. Thus an amplifier with high power handling capability $\left(P_{\text {sat }}\right)$ and IP3 (third order intercept point) has been used. After the second RF amplifier the RF signal is downmixed to the IF band ( $38 \mathrm{MHz})$. In order to implement coherent RF signal reception all the mixers in the system have to use the sample local oscillator signal, thus the RF power divider is used to distribute the local oscillator signal for the mixers of the four individual receiver channels. In the last stage the IF signal is filtered to suppress the RF and local signal leakage. The IF filter is also used as the anti-aliasing filter 
for the later digitalization step. It must be emphasized that all the components of the receiver system are wideband RF integrated circuits to ensure wideband software defined operation. The critical amplifier stages are MMICs (Monolithic Microwave Integrated Circuit) and the $\mathrm{RF}$ power divider is implemented as a resistive splitter.

\subsection{Digital Data Acquisition}

Fig. 2 highlights the structure of the digital data acquisition unit of the system.

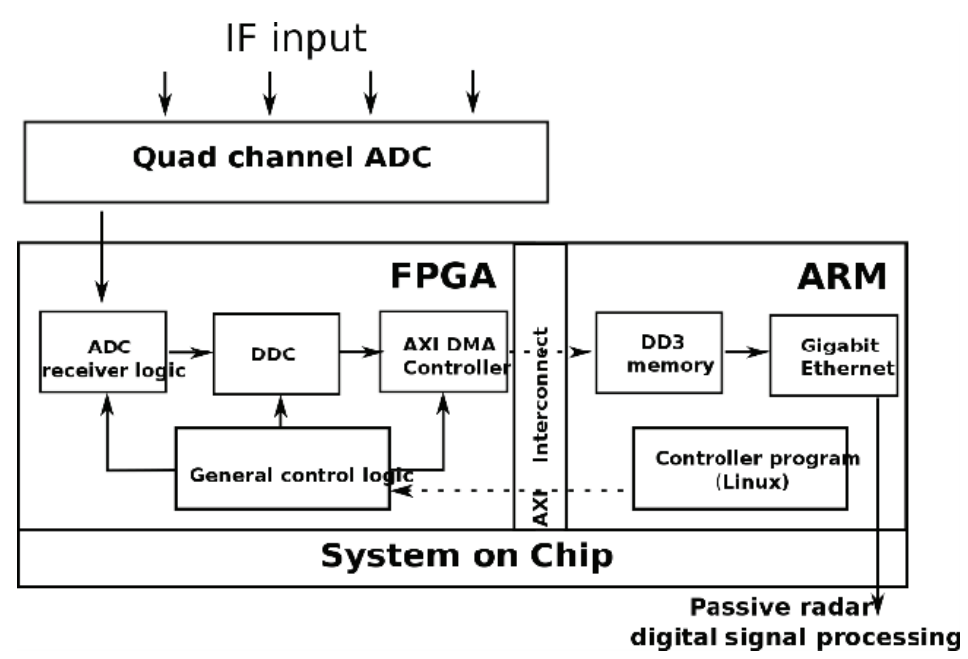

Fig. 2. Block diagram of the multichannel passive radar receiver platform

After the RF stage the IF signals are digitalized by the ADC which has serial data output. At this point of the system the data rate reach $100 \mathrm{MHz} 12$ bit $4=4.8 \mathrm{Gbit} / \mathrm{s}$. This high speed data stream is split into 8 different lines which greatly facilitates the transfer of the raw data. The transferred signals then arrive to the SoC (System on Chip). A picture from the receiver platform can be seen in Fig. 3.

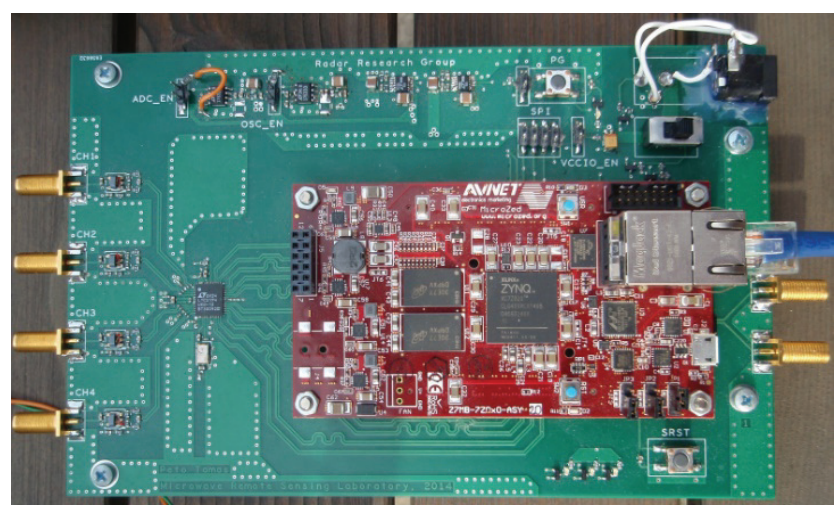

Fig. 3. Picture of the high speed digital data acquisition board 
We are using the Xilinx Zynq $7020 \mathrm{SoC}$ in the receiver platform. Application of SoC provides state of the art solution for mixed signal processing demands. The FPGA integrated into the chip is able to perform the critical signal processing tasks while the dual core ARM processor can handle effectively the algorithmic problems. Therefore, it is advisable to implement real time signal processing tasks with high data throughput in the FPGA logic. Accordingly, we have implemented the ADC receiver logic inside the PL (Programmable Logic). This logic is mainly responsible for the reception of the high speed raw data transferred by the ADC. The received digital samples are then forwarded to the DDC (Digital Down-Converter) circuit. The DDC circuit performs down-mixing to baseband, filtering, and decimation of the processed signal. Decimation ratio is determined according to the bandwidth of the currently used reference signal. During the presented experimental measurement, we used 12 as a decimation factor for the processing of the DVB-T signal which has $7.61 \mathrm{MHz}$ bandwidth. From the output of the DDC module the baseband IQ complex samples are forwarded to the DMA (Direct Memory Access) controller module. The main task of this logic is to move the data from the FPGA to the DDR3 memory used by the ARM processors with CPU offload. The Linux system running on the ARM processors transfers the received data through Gigabit Ethernet to a computer which performs the passive radar signal processing and detection algorithms. The flexibility and signal processing capability of the system relies on the effective information exchange between the two part of the system (PL-programmable logic and PSprocessing system). The operating system running on the PS manages the protocols needed to communicate with the outside world, while it is also able to reach the internal systems and registers of the PL directly. Because of this construction the passive radar receiver system can effectively change between the used reference sources by reconfiguring the internal parameters of the software defined radio blocks inside the system even during remote operation.

\section{Receiver demonstration results}

The wideband multichannel operation of the receiver has been tested with DVB-T transmission in the UHF band. To perform an adequate test for the beamforming capabilities, the effectiveness of the interference suppression has been analyzed as it is real demand in passive radar applications. The results of these measurement along with results of the DOA (Direction of Arrival) algorithms are presented.

\subsection{Calibration procedure}

Amplitude and phase response calibration of a multichannel receiver is essential for the proper and correct application of the beamforming techniques. In order to carry out relevant measurements, the receiver unit has been calibrated. This calibration procedure must be performed over the full frequency range of interest. For the presented demonstration the relative amplitude and phase responses of the individual channels have been recorded from $630 \mathrm{MHz}$ to $638 \mathrm{MHz}$ in the band of the used DVB-T transmission. 


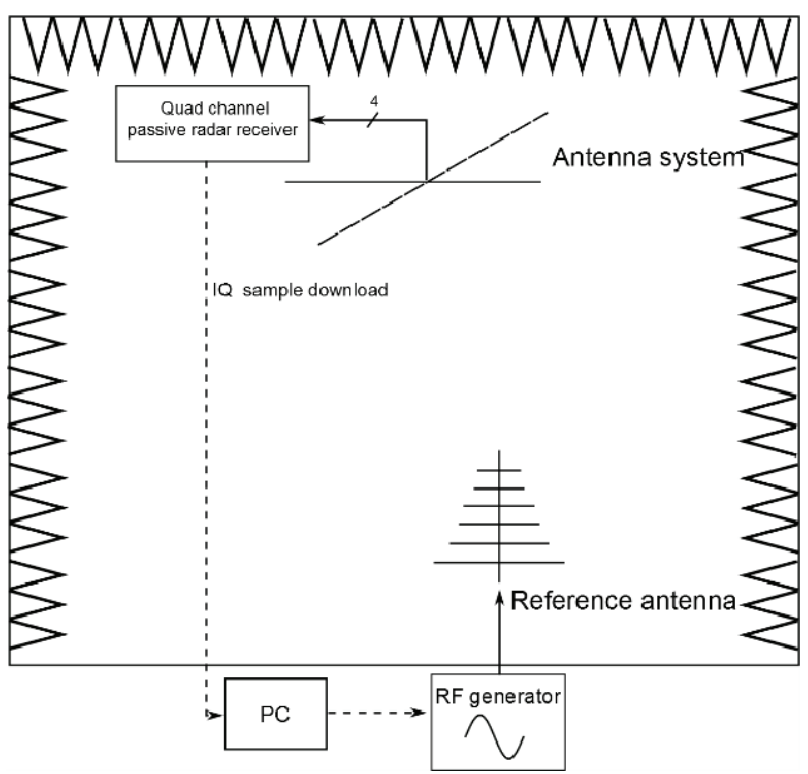

Fig. 4. Calibration measurement setup

The calibration has been performed in an anechoic chamber. The setup of this measurement is illustrated on Fig. 4. Both the RF generator and the receiver unit were, controlled from a PC. The RF CW (Continuous Wave) excitation signal had been set first, then the receiver downloaded sufficient IQ samples for the latter offline processing. For the reception of the RF signals we used an equidistant linear antenna array consisting of 4 fractal patch antenna elements with 0.52 wavelength $(634 \mathrm{MHz})$ distance between elements. As a reference antenna for the transmission of the RF calibration signal a wideband log-periodic antenna was used. The relative amplitude and phase difference between the channels was finally determined in frequency domain at the currently measured frequency. It has to be noted that in this calibration setup both the RF multichannel receiver chain and the antenna array were calibrated. While measuring the calibration matrix, the main direction of the antenna system was rotated precisely in the direction of the reference antenna. The arrangement of the measurement can be seen in Fig. 5.

Since the effective interference cancelation is crucial in passive radars applications, the performance of the calibration was determined with analyzing the suppression capabilities of the system. This has been carried out with the calculation of the averaged power before and after performing a beam space processing algorithm.

The success of the calibration has been tested first. During the test measurement the antenna system was rotated from its original calibration direction to a random direction to ensure more realistic scenario. The RF generator was programed to transmit a priori known wideband excitation signal in the same setup as in the calibration procedure. The IQ samples were recorded and then after correcting the samples the DOA of the test signal has been determined. For the DOA estimation Capon's method has been applied as it has high resolution. Eq. (4) describes the used Capon method. 


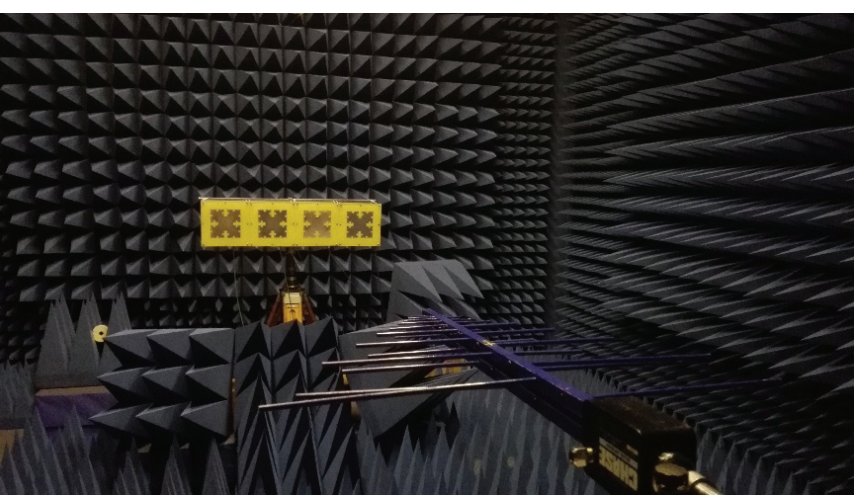

Fig. 5. Calibration measurement taken in the anechoic chamber

$$
P(\Theta)=\frac{1}{\boldsymbol{s}(\boldsymbol{\Theta})^{H} \boldsymbol{R}_{x x}{ }^{-1} \boldsymbol{s}(\boldsymbol{\Theta})},
$$

where $\boldsymbol{s}(\Theta)$ is the steering vector and $\boldsymbol{R}_{x x}$ is the spatial correlation matrix. Fig. 6 shows the calculated results.

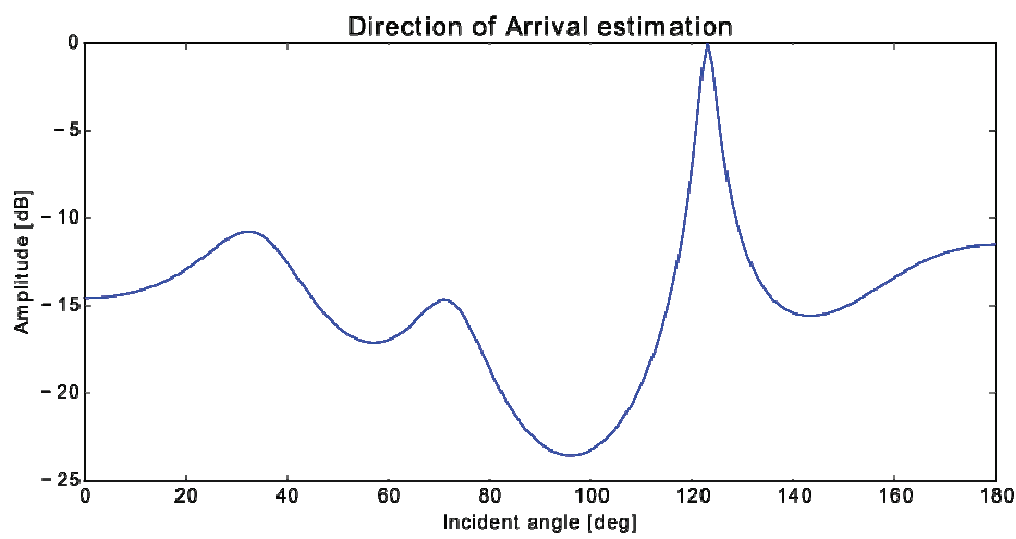

Fig. 6. Result of the DOA measurement taken in the anechoic chamber

From the result, the DOA of the test signal has been determined to $125 \mathrm{deg}$. On the basis of the knowledge on the incident angle of the transmitted test signal, the beampattern illustrated in Fig. 7 has been synthesized. The coefficient vector of the beamformer was calculated using the fixed MSIR (Maximum Signal to Interference Ratio) algorithm according to Eq. (5) which was configured to place wideband null in the direction of the interference signal.

$$
\boldsymbol{w}^{H}=\boldsymbol{u}^{H} \boldsymbol{A}^{-1} .
$$

In Eq. (5) $\boldsymbol{w}$ denotes the calculated coefficient vector of the beamformer, $\boldsymbol{u}$ means the constraint vector and $\boldsymbol{A}$ is the array response matrix. 


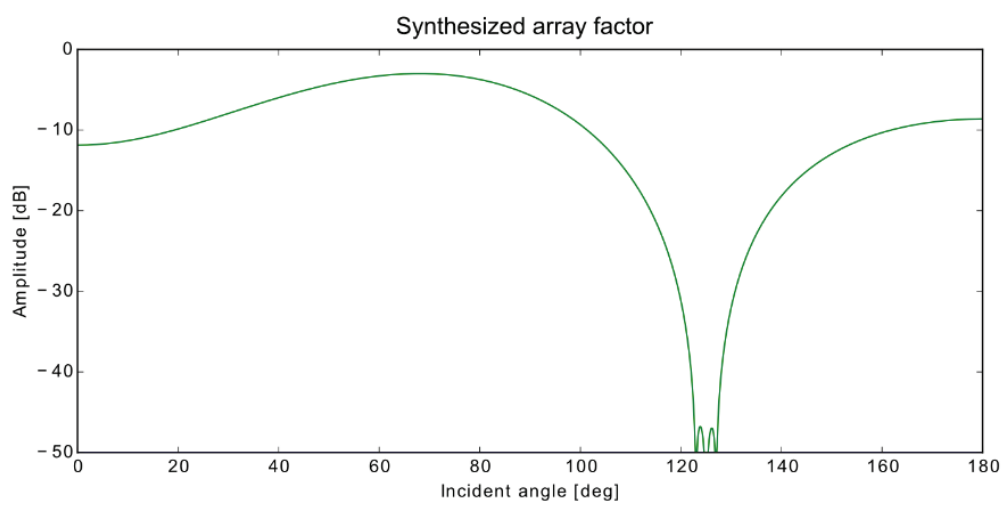

Fig. 7. Array factor of the synthesized radiation pattern for testing interference suppression in anechoic chamber

Finally, the cross correlation functions have been calculated to determine the power level of the known excitation signal. Fig. 8 shows the obtained results. The blue curve represents the autocorrelation function of the first receiver channel, while the red curve represents the cross-correlation function of the same receiver channel and the beamspace processed channel. It can be seen that the power level of the excitation signal has been effectively suppressed with $30 \mathrm{~dB}$.

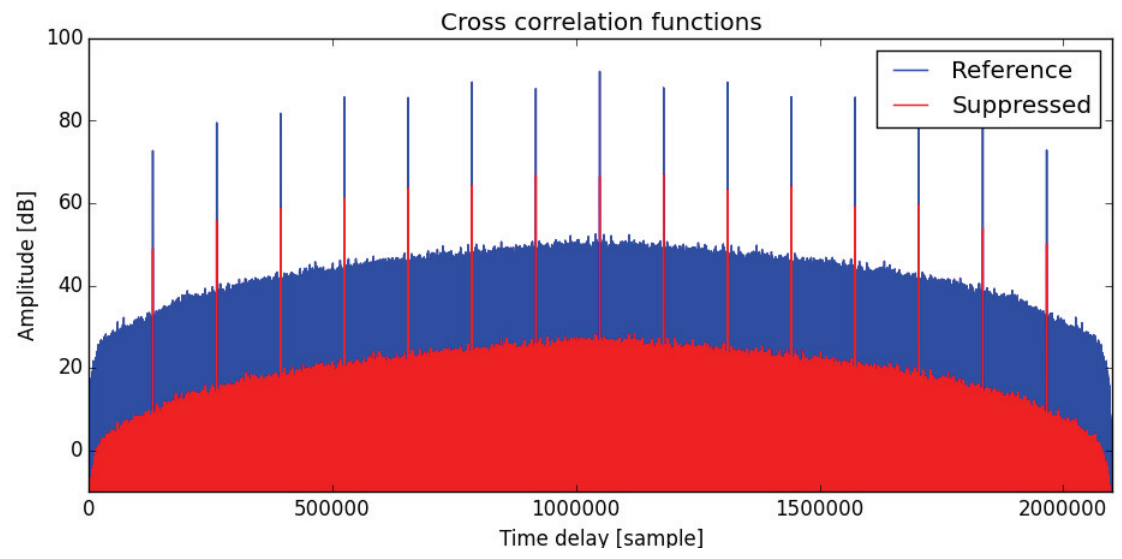

Fig. 8. Difference between cross correlation functions shows the power level reduction in the received signal

\subsection{Field measurements}

We have measured the beamforming capabilities of the system also in real environment using DVB-T reference signal. During the course of the experimental measurement we have first determined the exact positions of a transmitter tower applying Capon's direction of 
arrival algorithm and then we applied interference suppression technique in the same way as it was used in the calibration test.

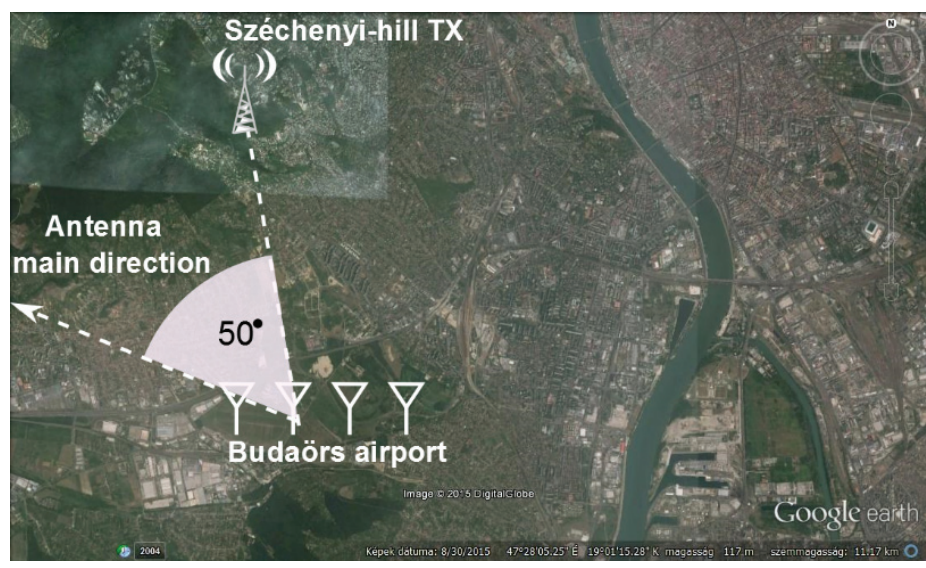

Fig. 9. Measurement scenario

For the measurement we used the signals of a DVB-T transmitter operating in Budapest. The transmitter tower is located at the Széchenyi-hill. The geometrical layout of the transmitters and the receiver system is depicted in Fig. 9. After the completion of the measurement, the downloaded IQ complex data was processed offline.

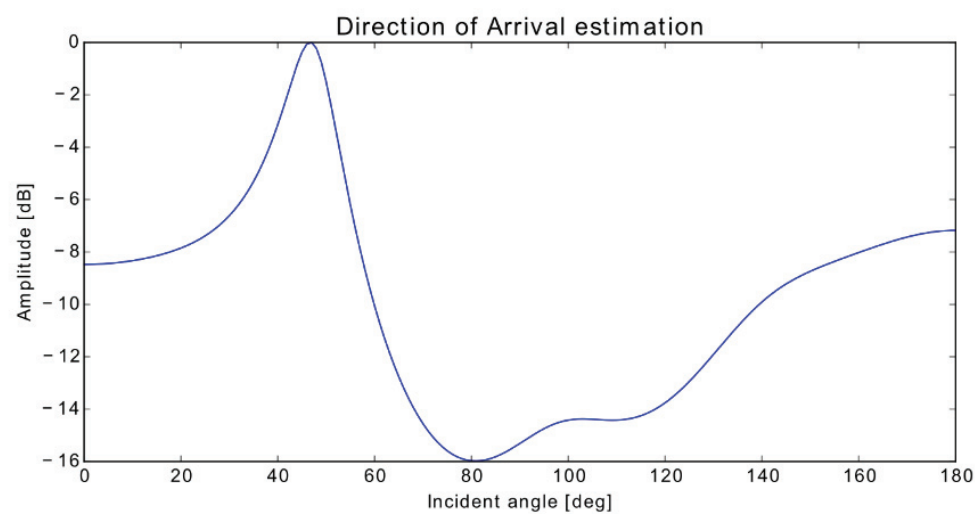

Fig. 10. Measured DOA of the DVB-T transmitter tower located at the Széchenyi-hill with Capon's method

Fig. 10 shows the results of the direction of arrival algorithm. Where, the function of the DOA estimation has its maximum at $48 \mathrm{deg}$. Based on the preliminary calculated transmitter direction we can conclude that the DOA of the DVB-T transmitter tower could be determined precisely. The results of the direction of arrival algorithms match with the expected results. After having measured the DOA of the reference signal, the antenna channels have been 
processed in order to produce the surveillance channel for the passive radar. The synthesized beampattern can be seen in Fig. 11. Nulls are placed in the direction of the DVB-T transmitter.

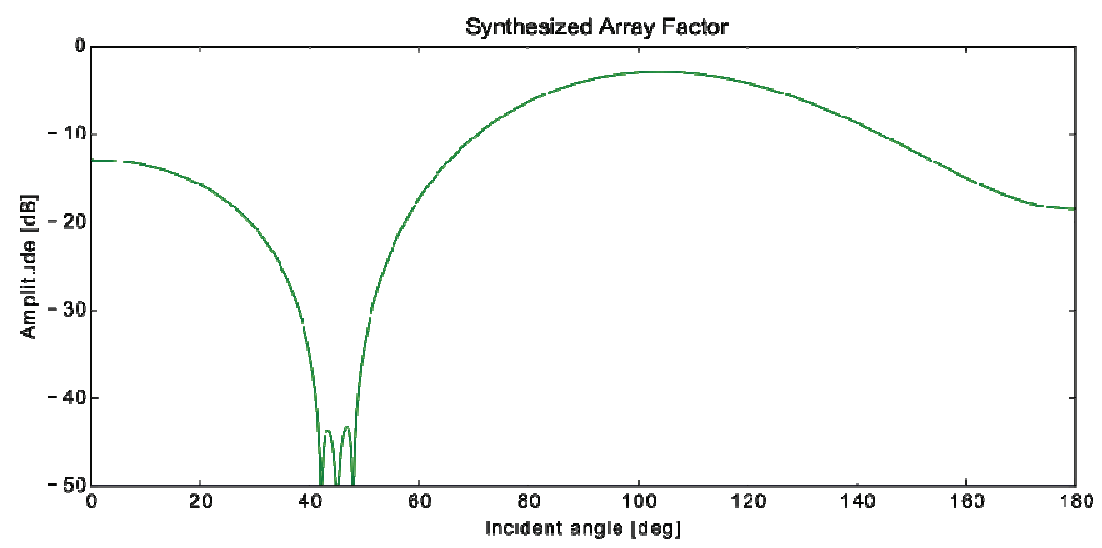

Fig. 11. Synthesized surveillance channel beampattern for the experimental measurement

Correlation functions are calculated finally to estimate the achieved DPIS (Direct Path Interference Suppression). The results are shown in Fig. 12. The reference curve shows the autocorrelation function of the first receiver channel, while the other curve represents the surveillance channel. Having examined the measurement results, $21 \mathrm{~dB}$ suppression has been achieved.

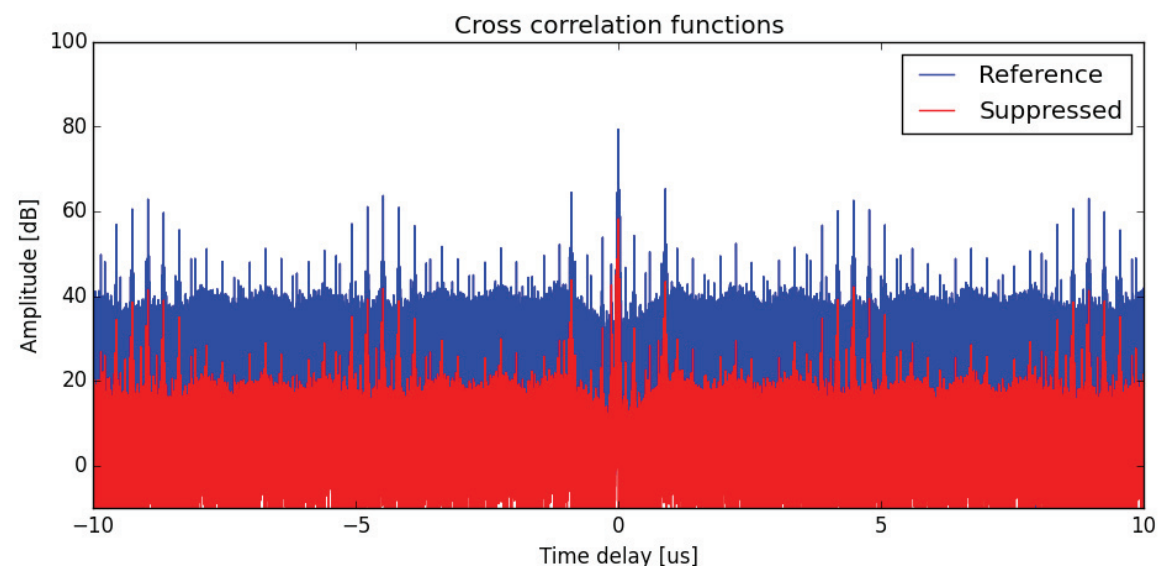

Fig. 12. Estimation of the achieved DPIS in the field measurement

Based on the presented measurement results it can be concluded that the developed multichannel receiver platform performs properly as expected. 


\section{Conclusions}

In order to widespread the passive radar technology it is necessary to develop such hardware supported systems that target the inherent advantages and difficulties of the passive radars. The saturation of the electromagnetic environment provides great opportunity to passive radars, however special receiver platforms are required. In this paper we presented the development of a passive radar receiver platform which applies modern system on chip architecture with high integrity, flexibility and signal processing capability. The operation of the receiver structure has been tested with the DVB-T signal. The performance of the multichannel operation including the DOA estimation, and the DPIS have been demonstrated. Further researches can be done to effectively implement high performance passive radar signal processsing algorithms utilizing the DSP capabilities of the SoC.

\section{Acknowledgements}

This work has been supported in the frame of the project "Multichannel passive ISAR imaging for military applications (MAPIS) No. B-1359 IAP2 GP" of the European Defence Agency.

\section{References}

[1] Jamil K., Alam M., M. Hadi A., Alhekail Z.O., A multi-band multi-beam software-defined passive radar part I: System design, IET International Conference on Radar Systems, Glasgow, United Kingdom, pp. 1-4 (2012).

[2] Alam M., Jamil K., Alhekail Z.O., Al-Humaidi S., A multi-band multi-beam software-defined passive radar part II: Signal processing, IET International Conference on Radar Systems, Glasgow, United Kingdom, pp. 1-5 (2012).

[3] Heunis S., Paichard Y., Inggs M., Passive Radar using a Software-Defined Radio Platform and Opensource Software Tools, IEEE Radar Conference 2011, Kansas City, United States of America, pp. 879-884 (2011).

[4] Wang Q., Hou C., Lu Y., An Experimental Study of WiMAX-Based Passive Radar, IEEE Transactions on Microwave Theory and Techniques, vol.58, no. 12, pp. 3502-3510 (2010).

[5] Tan D.K.P., Sun H., Lu Y., Liu W., Feasibility analysis of GSM signal for passive radar, IEEE Radar Conference 2003, Huntsville, United States of America, pp. 425-430 (2003).

[6] Peto T., Dudás L., Seller R., Péter Renner, Digital television broadcast-based passive radar research and development, 20th International Conference on Microwaves, Radar, and Wireless Communication (MIKON), Gdansk, Poland, pp. 1-4 (2014).

[7] Tao R., Wu H.Z., Shan T., Direct-path suppression by spatial filtering in digital television terrestrial broadcasting-based passive radar, IET Radar, Sonar \& Navigation, vol. 4, no. 6, pp. 791-805 (2010).

[8] Malanowski M., Kulpa K., Digital beamforming for Passive Coherent Location radar, IEEE Radar Conference 2008, Rome, Italy pp. 1-6 (2008).

[9] Moscardini C., Conti M., Berizzi F et al., Spatial Adaptive Processing for Passive Bistatic Radar, IEEE Radar Conference 2014, Cincinnati, United States of America, pp. 1061-1066 (2014).

[10] Conti M., Berizzi F., Martorella M. et al, High range resolution multichannel DVB-T passive radar, IEEE, vol. 27, no. 10, pp. 37-42 (2012). 